\title{
Effect of probiotics and selenium-containing preparations on nonspecific resistance of calves
}

\author{
Victor Eremenko ${ }^{1}$, Oleg Getokov 2,*, Boris Dzagurov ${ }^{3}$, Safarbi Kazanchev², and Murat \\ Ulimbashev $^{2}$ \\ ${ }^{1}$ Kursk State Agricultural Academy named after I.I. Ivanov, 70 Marksa Str., 305021 Kursk, Russia \\ ${ }^{2}$ Kabardino-Balkarian State Agricultural University named after V. M. Kokov, Lenin Avenue, 1V, \\ 360030 Nalchik, Russia \\ ${ }^{3}$ Gorsky State Agrarian University, 37 Kirova Str., 362040 Vladikavkaz, Russia
}

\begin{abstract}
Black-and-white Holstein calves were studied. 3 experimental groups were formed: they included calves of two weeks of age, 10 animals each. The calves of the first group received "Intestevit" probiotic with milk at the rate of 3 doses per day for 1 animal for 15 days. The second group of calves, in addition to "Intestevit" preparation, also received seleniumcontaining preparation "Selen Plex" ${ }^{\mathrm{TM}}$ " at a dose of $1 \mathrm{mg} / \mathrm{kg}$. The third group was the control group. Blood for testing was taken from the tail vein before the morning feeding and the start of preparation feeding, 30 and 60 days after the use of these preparations. Feeding "Intestevit" preparation to calves from 15 days of age for 60 days increases the following indicators relative to the control group: total immunoglobulins by $8.4 \%$; Leukocyte phagocytic activity (LPA) - 10.5\%; serum lysozyme activity (SLA) $20.1 \%$; serum bactericidal activity (SBA) - 4.6\%; T-lymphocytes (absolute content) - 14.1\%; B-lymphocytes (absolute content) - 1.5\%. Feeding "Intestevit + Selen Plex ${ }^{\mathrm{TM}}$ " complex preparation to calves from 15 days of age for 60 days increases the following indicators relative to the control group: total immunoglobulins by $11.7 \%$; LPA - $12.1 \%$; SLA - $15.8 \%$; SBA - 7.6\%; T-lymphocytes (absolute content) - 17.8\%; B-lymphocytes (absolute content) $-2.2 \%$.
\end{abstract}

\section{Introduction}

It is not possible to consider all aspects to satisfy animal boy needs in industrial animal husbandry. It results in various diseases and a decrease in productivity [1-5]. In this regard, an increase in the level of productivity of animals, their health will be associated with satisfaction of their physiological need for nutrients to contribute to implementation of their genetic potential. To solve this problem, the most intense ontogenesis periods should be considered, when the future productivity of animals is formed. The most vulnerable is the immune system, highly affected by various factors [6-8]. Therefore, it is necessary to search for the most effective methods to enhance the body resistance, especially in the early

${ }^{*}$ Corresponding author: getokov777@mail.ru 
period of ontogenesis. One of these methods is the use of probiotic and selenium-containing preparations in diets [9-14]. The main advantage of probiotic preparations is the absence of side effects and harmlessness to the animal body and resulting products. Probiotic preparations balance the intestinal microbiocenosis and provide a beneficial effect on the animal body as a whole. Selenium-containing preparations are also used in animal husbandry. Selenium is part of the enzyme glutathione peroxidase. Therefore, seleniumcontaining preparations help to reduce the activity of lipid peroxidation and increase antioxidant protection. One of these selenium-containing preparations is Selen Plex ${ }^{\mathrm{TM}}$ : it is a selenium protein. According to its effect on the body, it is non-toxic, compared to inorganic selenium preparations.

Selenprotein has more pronounced antioxidant properties. Therefore, the study of selenium-containing and probiotic preparations effect on the specific resistance of growing calves is relevant.

Study Purpose. The study purpose was to analyze the state of nonspecific resistance in growing calves when using "Intestevit" probiotic and selenium-containing "Selen Plex TM" preparation in their diet.

\section{Study methods}

Black-and-white Holstein calves were studied. 3 experimental groups were formed: they included calves of two weeks of age, 10 animals each. The calves of the first group received "Intestevit" probiotic with milk at the rate of 3 doses per day for 1 animal for 15 days. The second group of calves, in addition to "Intestevit" preparation, also received selenium-containing preparation "Selen Plex ${ }^{\mathrm{TM}}$ " at a dose of $1 \mathrm{mg} / \mathrm{kg}$. The third group was the control group. The conditions for keeping and feeding the calves were identical. Blood for testing was taken from the tail vein before the morning feeding and the start of preparation feeding, 30 and 60 days after the use of these preparations. In blood samples, total immunoglobulins were determined by the zinc sulfate method using a turbidity reaction. T-lymphocytes, B-lymphocytes, phagocytic activity of leukocytes, phagocytic index, phagocytic number were studied according to V.S. Gostev. Lysozyme activity of blood serum was determined using Micrococcus lisodeikticus culture, and bactericidal activity of blood serum using Staphylococcus aureus culture.

The obtained study results were subjected to biometric processing.

\section{Study results}

\subsection{Total immunoglobulins}

The concentration of total immunoglobulins in the blood of experimental calves is shown in Figure 1.

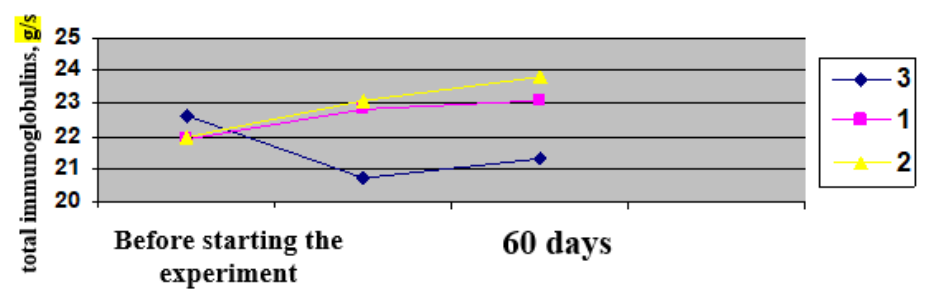

Fig. 1. Concentration of total immunoglobulins in the blood of experimental calves. 
The presented data show that before using the preparations in all three groups, the level of this indicator was practically the same and amounted to $21.6 \pm 0.4 \mathrm{~g} / \mathrm{l}$ in the control group, $21.9 \pm 0.41$ in the first experimental group, in the second experimental group $22.0 \pm$ 0.5 1. 30 days after the start of "Intestevit" administration, the level of total immunoglobulins increased to $22.8 \pm 0.5 \mathrm{~g} / \mathrm{l}$, and in the group of calves that received Intestevit + Selen Plex ${ }^{\mathrm{TM}}$ preparations, $23.1 \pm 0,3 \mathrm{~g} / \mathrm{l}$. Then, with increasing age of calves, 2 months after the start of administration, the level of total immunoglobulins continued to increase in both experimental groups. The calves that received "Intestevit", after 60 days in their blood, the concentration of total immunoglobulins in comparison with the control group was higher by $1.8 \mathrm{~g} / 1$ and amounted to $23.1 \pm 0.4 \mathrm{~g} / \mathrm{l}$. In the group of calves that received "Intestevit" + "Selen Plex TM", after 60 days the level of total immunoglobulins was $2.5 \mathrm{~g} / 1$ higher than in the control group and amounted to $23.8 \pm 0.4 \mathrm{~g} / \mathrm{l}$. In the control group, the concentration of total immunoglobulins was at a lower level relative to similar data of experimental groups.

\subsection{Leukocyte phagocytic activity}

The performed studies of leukocyte phagocytic activity are shown in Figure 2.

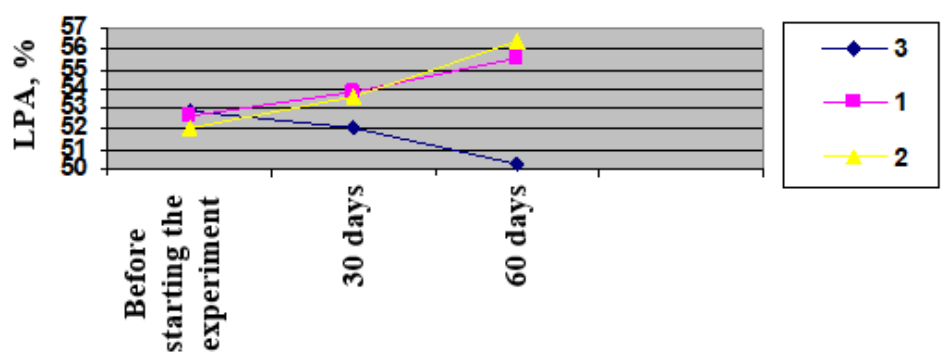

Fig. 2. Leukocyte phagocytic activity in the blood of experimental calves.

Analysis of the results shows that the cellular protection of blood in the calves of experimental groups was relatively more pronounced relative to the control group, where they did not receive preparations. Before using preparations in the control group, LPA was $52.9 \pm 1.8 \%$. In compared groups, this indicator was approximately the same and amounted to $52.6 \pm 1.2 \%$ and $52.0 \pm 1.7 \%$. After 30 days from the beginning of the "Intensiveit" preparation, LPA was $53.9 \pm 1.6 \%$, and in the group of calves that received "Intestevit + Selen Plex ${ }^{\mathrm{TM}}$ " preparations - 53.6 $\pm 1.1 \%$. In the subsequent period of the experiment, LPA in both experimental groups increased. In the group of calves that received "Intestevit", after 60 days, LPA was at the level of $55.6 \pm 1.3 \%$, and in the group of calves that received "Intestevit + Selen Plex" ${ }^{\mathrm{TM}}$ " $56.4 \pm 1.2 \%$. The increase relative to the data before administration was by $5.7 \%$ and $8.4 \%$, respectively, by groups. It proves that the complex use of "Intestevit + Selen Plex ${ }^{\mathrm{TM} "}$ preparations has a more significant effect on the blood cellular defense. During the experiment, the phagocytic index (PI) had the same direction of change as the leukocyte phagocytic activity, Figure 3. 


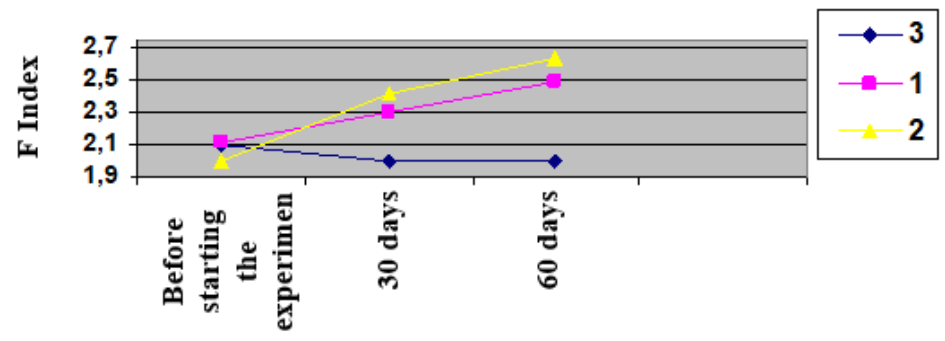

Fig. 3. Phagocytic blood index of experimental calves.

So, in the period before administration in the control group, the phagocytic blood index was $2.1 \pm 0.08$ and in subsequent periods did not change and remained at the same level. In experimental groups, it was subject to changes in the direction of increase. So, in the group of calves that received "Intensive" after 30 and 60 days from the beginning of admission, it was $2.31 \pm 0.11$ and $2.49 \pm 0.12$, respectively. Regarding the data before administration, this increase by the 60 th day was $18 \%$. In the group of calves that received complex preparation "Intestevit + Selen Plex ${ }^{\mathrm{TM}}$ ", the dynamics of changes in this preparation was as follows: So, after 30 and 60 days, the phagocytic index was $2.42 \pm 2.10$ and $2.63 \pm 0.14$. Regarding the data before administration, this increase by the 60 th day was $31.5 \%$.

Lysozyme and bactericidal activity of blood serum play a significant role in the natural resistance formation.

\subsection{Serum lysozyme activity (SLA)}

The study results of serum lysozyme activity are shown in Figure 4.

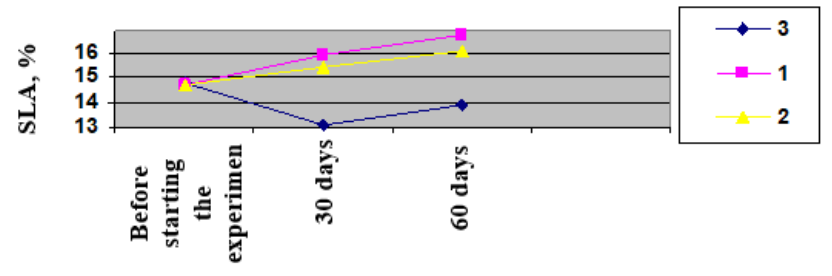

Fig. 4. Serum lysozyme activity of experimental calves

The above data demonstrate that before the start of administration in experimental and control groups, SLA was the same and within the range of $14.7-14.8 \%$. In subsequent experimental periods in the control group, it decreased and was $13.1 \pm 0.20 \%$ and $13.9 \pm 0.20 \%$ on days 30 and 60 , respectively. In experimental groups, on the contrary, this indicator increased after administration of preparations. So, after 30 days and 60 days following the start of "Intestevit" administration SLA was $15.9 \pm 0.18 \%$ and $16.7 \pm 0.19 \%$, respectively. In the experimental group, where calves received "Intestevit + Selen Plex ${ }^{\text {TM" }}$ complex preparation, these values after 30 and 60 days were $15.4 \pm 0.18 \%$ and $16.1 \pm 0.19 \%$.

\subsection{Serum bactericidal activity (SBA)}

Before the experiment, SBA was slightly higher in the control group of calves and amounted to $43.5 \pm 2.1 \%$, Figure 5 . 


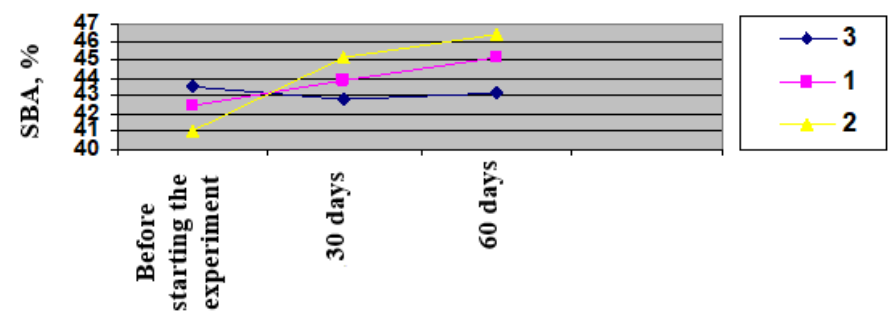

Fig. 5. Serum bactericidal activity of experimental calves

In compared groups, this indicator amounted to $42.4 \pm 2.4 \%$ and $41.0 \pm 1.3 \%$. In the control group, in the subsequent periods of the experiment after 30 and 60 days, this indicator was $42.8 \pm 2.2 \%$ and $43.1 \pm 2.0 \%$. In calves that received "Intestevit" preparation, SBA, 30 and 60 days after the start of administration, was $43.9 \pm 1.7 \%$ and $45.1 \pm 2.0 \%$, respectively. In the ccompared experimental group, where calves received "Intestevit + Selen Plex ${ }^{\mathrm{TM}}$ " complex preparation, these values after 30 and 60 days were $45.1 \pm 1.1 \%$ and $46.4 \pm 1.2 \%$. These values were higher than in the group of calves that received "Intestevit".

\subsection{T-lymphocytes}

The results of the relative content of T-lymphocytes are shown in Figure 6.

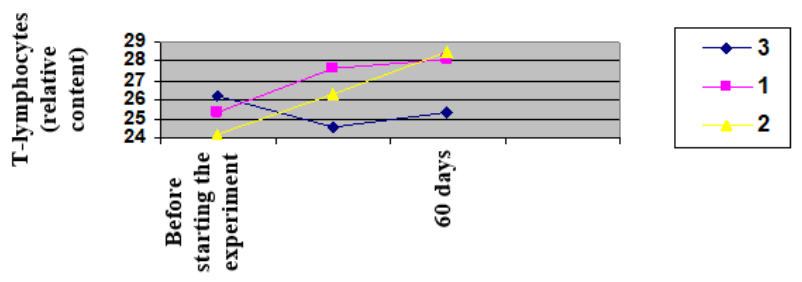

Fig. 6. The content (relative) of T-lymphocytes in the blood of experimental calves.

As the above data show, the absolute and relative content of T-lymphocytes in experimental calves that received "Intestevit" and "Intestevit + Selen Plex ${ }^{\mathrm{TM}}$ " preparations was higher than in the control group. Before testing, the relative content of T-lymphocytes in all groups was approximately the same. So in the control group it was $25.2 \pm 1.1 \%$, in the group that received "Intestevit + Selen Plex" ${ }^{\mathrm{TM}}$ " $-24.2 \pm 1.1 \%$. The maximum level of Tlymphocytes in experimental groups reached 60 days after administration. In the group of calves that received "Intestevit", the relative content after this interval of "Intestevit" administration was $28.1 \pm 1.0 \%$, and in the group that received "Intestevit + Selen Plex" TM" complex preparation $-28.5 \pm 1.1 \%$. The absolute content of T-lymphocytes is shown in Figure 7.

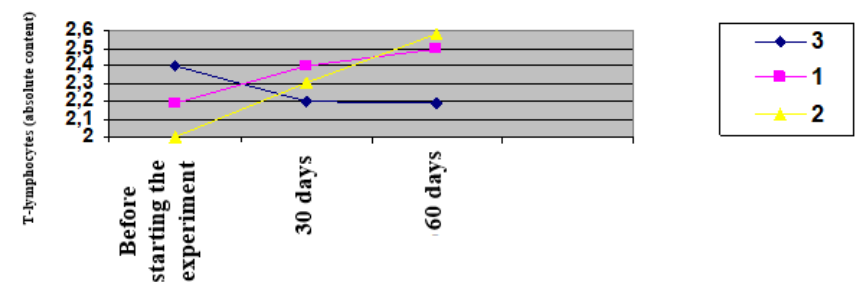

Fig. 7. The content (absolute) of T-lymphocytes in the blood of experimental calves. 
From the above data it is obvious that in the control group with increasing age of calves, the absolute content of this indicator decreased from $2.4 \pm 0.11 \cdot 10^{9} / 1$ to $2.19 \pm 0.08 \cdot 10^{9} / 1$. In the group of cow calves that received "Intestevit", the absolute content of T-lymphocytes increased from $2.19 \pm 0.12 \times 10^{9} / 1$ to $2.50 \pm 0.09 \times 10^{9} / 1$, in the group that received "Intestevit + Selen Plex ${ }^{\mathrm{TM}}$ " complex preparation it increased from $2.0 \pm 0.12 \times 10^{9} / 1$ to $2.58 \pm 0.14 \mathrm{x}$ $10^{9} / 1$.

\subsection{B-lymphocytes}

Figure 8 shows the study results of the relative content of B-lymphocytes.

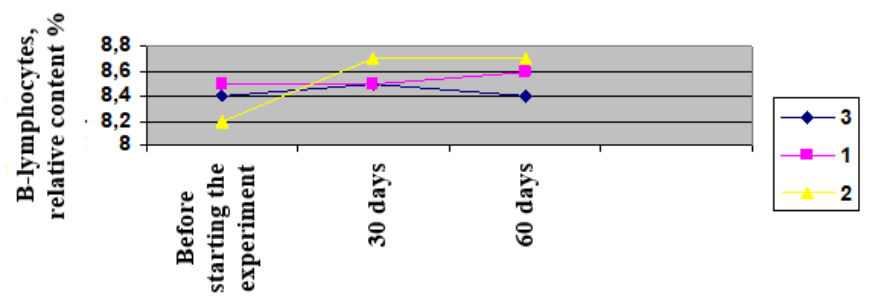

Fig. 8. The content (relative) of B-lymphocytes in the blood of experimental calves.

These data prove that in control group animals the relative content of B-lymphocytes did not change with increasing age and amounted to $8.4 \%$, the absolute content changed from $1.35 \pm 0.08$ to $1.33 \pm 0.09 \cdot 10^{9} / 1$. Figure 9. In the group of calves that received "Intestevit" preparation, there were practically no changes in the relative and absolute content of B-lymphocytes. So, the relative content was within the range of $8.5 \pm 0.13$ $8.6 \pm 0.15 \%$, and the absolute $1.34 \pm 0.09-1.35 \pm 0.08 \cdot 10^{9} / 1$.

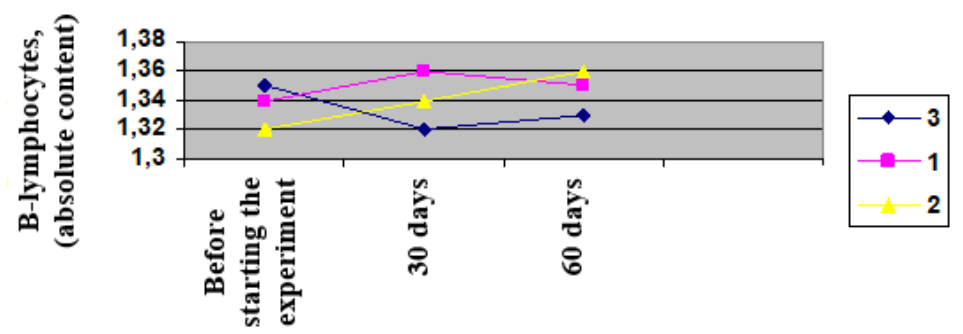

Fig. 9. The content of B-lymphocytes (absolute) in the blood of experimental calves.

The increase in the relative and absolute value of B-lymphocytes occurred in the group of calves that received "Intestevit + Selen Plex TM" preparation. Thus, the relative content of B-lymphocytes increased from $8.2 \pm 0.5$ to $8.7 \pm 0.18 \%$, the absolute content from $1.32 \pm 0.08$ to $1.36 \pm 0.10 \times 10^{9} / 1$.

\section{Conclusions}

1. Feeding "Intestevit" preparation to calves from 15 days of age for 60 days increases the following indicators relative to the control group: total immunoglobulins by $8.4 \%$; Leukocyte phagocytic activity (LPA) - 10.5\%; serum lysozyme activity (SLA) - $20.1 \%$; serum bactericidal activity (SBA) - 4.6\%; T-lymphocytes (absolute content) - 14.1\%; Blymphocytes (absolute content) - 1.5\%. 
2. Feeding "Intestevit + Selen Plex ${ }^{\mathrm{TM}}$ " complex preparation to calves from 15 days of age for 60 days increases the following indicators relative to the control group: total immunoglobulins by $11.7 \%$; LPA - 12.1\%; SLA - 15.8\%; SBA - 7.6\%; T-lymphocytes (absolute content) - 17.8\%; B-lymphocytes (absolute content) - 2.2\%.

\section{References}

1. M. Averenkova, Problems of Veterinary Sanitation, Hygiene and Ecology, 118, 190 (2006)

2. T. D. Buzanova, Abstract of Thesis of Master of Vet. Sciences, 22 (2007)

3. N. V. Burnysheva, Abstract of a Thesis of Master of Agriculture, 22 (2007)

4. V. I. Eremenko, O. B. Sein, Metabolic status. Nonspecific resistance and their correlation in cattle (2011)

5. V. I., Eremenko, O. B. Sein, A. V. Titova, O. A., Prelikov, M. A. Blednov, Zootechnics, 7, 27 (2009)

6. Ya. Slabitskyi, S. Voloshanskaya, Agricultural Biology, 1, 21 (1987)

7. G. I. Boryaev, Abstract of a Thesis of Doctor of Biological Sciences, 43 (2000)

8. A. Bedenko, Milk and Feed. Management, 4, 32 (2007)

9. V. V. Ermakov, V. V. Kovwalskiy, Biological Significance of Selenium (1974)

10. N. G. Ignatiev, N. K. Kirillov, Veterinarian, 4, 55 (2001)

11. B. Seremak, Folia Univ. agr. Stetin. Zoctechn, 37, 63 (1999)

12. L. Tucker, Jnt. Hatchery Pract, 21, 7 (2007)

13. J. Kruze, H. Ceballos, A. Stryhn, A. Mella et al, Vet. Med., 9(54), 478 (2007)

14. T. D. Buzanova, Abstract of a Thesis for Master of Vet. Sciences, 22 (2007) 